\title{
COMPORTAMENTO HIGROSCÓPICO DA FARINHA DE PUPUNHA (Bactris gasipaes) ${ }^{1}$
}

\author{
Cristiane D. FERREIRA ${ }^{2}$, Rosinelson S. PENA ${ }^{2, *}$
}

\section{RESUMO}

Avaliou-se o comportamento higroscópico da farinha de pupunha obtida por secagem em estufa a $70^{\circ} \mathrm{C}$. Obteve-se dados de equilíbrio de adsorção de umidade a $15^{\circ} \mathrm{C} \mathrm{e} 35^{\circ} \mathrm{C}$, através de um método estático, onde recipientes de $500 \mathrm{~mL}$, contendo soluções saturadas de sais, foram utilizados como ambientes geradores de umidades relativas constantes, na faixa de $11 \%$ a $97 \%$, aos quais foram submetidas amostras do produto. A partir dos dados de equilíbrio foram construídas isotermas de adsorção de umidade, sendo observadas isotermas do tipo III e, ainda, que a umidade de equilíbrio diminui com o aumento da temperatura. Determinou-se o calor isostérico de adsorção para diferentes niveis de umidade de equilíbrio e a umidade equivalente à monocamada, observando-se valores inferiores a $5 \mathrm{~g} \mathrm{H}_{2} \mathrm{O} / 100$ g s.s. Verificou-se a aplicabilidade do modelo GAB na predição de dados de equilíbrio do produto. De acordo com os resultados obtidos, a farinha de pupunha apresenta baixa higroscopicidade, ou seja, baixa afinidade por moléculas de água.

Palavras-chave: atividade de água; umidade; ajustes; calor de adsorção.

\section{SUMMARY}

HYGROSCOPIC BEHAVIOR OF THE PUPUNHA FLOUR (Bactris gasipaes). It was studied the hygroscopic behavior of the pupunha flour obtained by drying in the oven at $70^{\circ} \mathrm{C}$. It was collected data of moisture adsorption for two temperatures $\left(15^{\circ} \mathrm{C}\right.$ and $\left.35^{\circ} \mathrm{C}\right)$, using the static method, where the recipients with the capacity of $500 \mathrm{~mL}$, having saturated salt solutions, it was used as environment generators of constant relative humidity, ranging from $11 \%$ to $97 \%$, which were submitted the samples of the product. From the equilibrium data, it was built isotherms of moisture adsorption, being observed type III isotherms, but even that the equilibrium moisture for the product decreases with the temperature increase. It was determined the isosteric heat of adsorption for different levels of equilibrium moisture and the equivalent moisture to the monolayer, which were inferior of $5 \mathrm{~g} \mathrm{H}_{2} \mathrm{O} / 100 \mathrm{~g} \mathrm{~s}$.s. It was verified the application of the GAB model in the equilibrium data prediction for the product. According to the results obtained, the pupunha flour shows low hygroscopicity, low affinity for water molecule.

Keywords: water activity; moisture; fitting; adsorption heat.

\section{1 - INTRODUÇÃO}

A pupunheira (Bactris gasipaes) é uma palmeira nativa dos trópicos úmidos americanos, e segundo CLEMENT [5] é a única espécie da família Palmae. Produz frutos comestiveis de sabor muito apreciado, definitivamente integrados aos hábitos alimentares da Região Amazônica [17]. A polpa dos frutos, cuja composição média é apresentada na Tabela 1, é geralmente consumida após cozimento em água e sal, mas pode ser utilizada na fabricação de farinhas para usos variados [7].

TABELA 1. Composição de diferentes tipos de pupunha [6].

\begin{tabular}{ccccccc} 
& \multicolumn{7}{c}{$\%$ em base seca } \\
Tipo & Umidade & Proteinas & Lipidios & Fibras & Cinzas & Carboidratos \\
\hline 1 & 55,7 & 23,0 & 6,9 & 9,3 & 1,3 & 59,7 \\
2 & 49,9 & 11,5 & 9,8 & 2,8 & 2,4 & 73,7 \\
3 & 56,7 & 8,3 & 6,1 & 3,6 & 2,1 & 79,9 \\
4 & 61,8 & 8,7 & 8,0 & 0,9 & 1,4 & 81,0 \\
5 & 58,0 & 12,9 & 7,7 & 0,9 & 1,6 & 77,8 \\
6 & 56,1 & 18,1 & 6,9 & 0,9 & 1,4 & 72,3 \\
7 & 53,2 & 16,1 & 8,4 & 0,9 & 1,4 & 72,2 \\
8 & 57,1 & 13,3 & 9,1 & 0,8 & 1,5 & 75,3 \\
\hline
\end{tabular}

Para obtenção da farinha de pupunha, utiliza-se a polpa dos frutos sem as cascas e caroços. A polpa, em-

1. Recebido para publicação em 21/02/2002. Aceito para publicação em 06/01/2003 (000806).

2. DEQAL/UFPA - Campus do Guamá - 66075-900 - Belém (PA) Tel.(91)2111297-rspena@ufpa.br

* A quem a correspondência deve ser enviada. bora rica em gordura, é suficientemente amilácea para permitir o preparo da farinha. Análises fisico-quimicas comprovam que a farinha de pupunha é um alimento de alto valor energético (Tabela 2).

TABELA 2. Composição de farinhas de diferentes tipos de pupunha [7].

\begin{tabular}{lcccccc}
\multicolumn{1}{c}{ Constituinte } & \multicolumn{7}{c}{ Tipo } \\
\cline { 2 - 8 } & $\mathbf{1}$ & $\mathbf{2}$ & $\mathbf{3}$ & $\mathbf{4}$ & $\mathbf{5}$ & $\mathbf{6}$ \\
\hline Umidade \% & 8,4 & 7,2 & 7,4 & 8,0 & 8,7 & 9,3 \\
Proteína \% & 6,6 & 4,1 & 5,3 & 5,4 & 5,2 & 4,9 \\
Extrato etéreo \% & 11,2 & 11,2 & 11,7 & 10,6 & 8,9 & 22,4 \\
Fibras \% & 1,3 & 1,6 & 1,1 & 1,7 & 1,9 & 2,4 \\
Açúcares redutores \% & 5,6 & 5,9 & 4,8 & 4,9 & 4,9 & 3,8 \\
Açúcares totais \% & 8,8 & 8,5 & 7,4 & 7,4 & 6,4 & 6,5 \\
Carotenóides totais* & 7,4 & 9,2 & 1,7 & 9,8 & 9,8 & 6,8 \\
* mg/100g de produto & & & & & &
\end{tabular}

A água é um dos principais componentes dos alimentos. Mesmo em produtos desidratados, como a farinha, onde ocorre em baixas proporções, é um dos mais importantes, influenciando bastante sobre as características de armazenamento [21].

A umidade de um alimento é expressa pelo valor obtido na determinação da água total contida no mesmo. Entretanto, esse valor não fornece indicações de como a água está distribuída, bem como não permite saber se toda ela está ligada da mesma maneira. Desta forma, o conhecimento da distribuição da água no alimento é mais importante que simplesmente o conhecimento da umidade. 
A atividade de água $\left(\mathrm{a}_{\mathrm{w}}\right)$ é uma medida da quantidade de moléculas de água livres ou ativas, geralmente obtida em relação à pressão de vapor da água pura. Esta medida é de fundamental importância, visto que, por meio dela, podem ser previstas reações químicas e enzimáticas, e desenvolvimento de microrganismos. A partir do conhecimento da $\mathrm{a}_{\mathrm{w}}$ pode-se, também, propor sistemas adequados de embalagem para um produto [4, 25].

Isotermas de adsorção de umidade descrevem a quantidade de água adsorvida por um produto, em dada temperatura, em função da $\mathrm{a}_{\mathrm{w}}$ ou da umidade relativa [8]. Através das isotermas pode-se determinar a monocamada, a qual além de estar relacionada com o início de uma série de reações químicas de deterioração dos alimentos, representa o ponto de maior gasto de energia em um processo de secagem [11, 13, 20, 22].

O objetivo do trabalho é elaborar e caracterizar uma farinha, obtida a partir de uma variedade de pupunha regional e, em seguida levantar dados de equilibrio de adsorção de umidade, para estudar o comportamento higroscópico da mesma.

\section{2 - MATERIAL E MÉTODOS}

\section{1 - Matéria-prima}

A pupunha utilizada foi adquirida nas feiras livres de Belém (PA).

\subsection{1 - Caracterização física da pupunha}

Determinou-se: diâmetro, comprimento e peso dos frutos, através da média dos valores obtidos para 50 frutos escolhidos aleatoriamente, e a composição em casca, polpa e sementes, a partir das massas totais oriundas dos mesmos frutos.

\subsection{2 - Caracterização química da pupunha e da farinha}

$\mathrm{Na}$ caracterização química da pupunha e da farinha determinaram-se os teores de umidade, lipidios, proteínas, fibras, resíduo mineral fixo (cinzas) e carboidratos, de acordo com metodologia proposta pelo INSTITUTO ADOLFO LUTZ [9].

\section{2 - Obtenção da farinha de pupunha}

A rotina utilizada na obtenção da farinha está representada na Figura 1.

Frutos selecionados com base na uniformidade, não existência de manchas e ausência de deterioração, foram submetidos à lavagem com água corrente, branqueados com água em ebulição durante 40 minutos, sendo, em seguida, retirados cascas e caroços. A secagem foi realizada em estufa com ar parado à $70^{\circ} \mathrm{C}$, por 24 horas. $\mathrm{O}$ produto foi submetido à moagem, seguida de peneiramento.

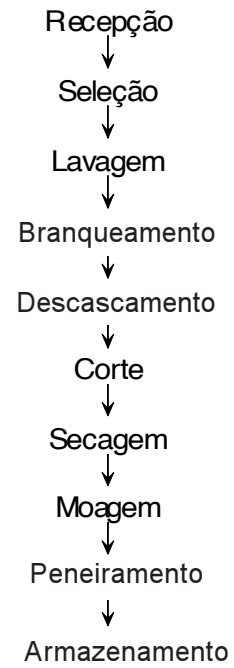

FIGURA 1. Rotina básica utilizada na obtenção da farinha de pupunha.

\section{3 - Obtenção das isotermas de adsorção}

O método utilizado na obtenção dos dados de equilíbrio de adsorção foi uma modificação do método estático dos dessecadores, com umidade relativa constante, sob vácuo, proposta por KIMURA E MAEDA [12] e testada por PENA [18].

Para obter as umidades relativas constantes foram utilizadas soluções saturadas de sais, preparadas de acordo com PENA et al [19]. As umidades relativas geradas pelas soluções saturadas, nas diferentes temperaturas, foram extraídas de ROCKLAND [23].

Amostras da farinha de pupunha dispostas em recipientes de polietileno e com aproximadamente $1 \mathrm{~g}$, após secagem em estufa a vácuo a $70^{\circ} \mathrm{C}$ [1], foram acondicionadas nos potes contendo as soluções saturadas. A cada ambiente foi aplicado vácuo de $127 \mathrm{~mm} \mathrm{Hg}$, transferindo-os, em seguida, para uma estufa incubadora para $\mathrm{DBO}$, nas temperaturas de $15^{\circ} \mathrm{C}$ e $35^{\circ} \mathrm{C} \pm 1^{\circ} \mathrm{C}$.

O processo foi acompanhado por pesagem das amostras a cada 24 horas, até o equilibrio. Durante este período as amostras foram submetidas à inspeção visual, para detectar quaisquer alterações perceptiveis. Todos os ensaios foram realizados em duplicata.

\section{4 - Predição das isotermas de adsorção}

O modelo matemático proposto por GuggenheimAnderson-De Boer (GAB) [16] (equação 1) foi ajustado aos dados de equilíbrio de adsorção, por regressão não linear, com auxilio do aplicativo STATISTICA for Windows 5.1B.

$$
\frac{m}{m_{o}}=\frac{c \cdot k \cdot a_{w}}{\left[\left(1-k \cdot a_{w}\right)\left(1-k \cdot a_{w}+c \cdot k \cdot a_{w}\right)\right]}
$$

onde: $\mathrm{m}$ é umidade; $\mathrm{a}_{\mathrm{w}}$ atividade de água; $\mathrm{m}_{\mathrm{o}}$ umidade na monocamada; c constante de Guggenheim; $\mathrm{k}$ fator relacionado as multicamadas. 


\section{5 - Determinação do calor isostérico de adsorção}

O calor isostérico integral de adsorção $\left(Q_{\mathrm{st}}\right)$ foi determinado utilizando-se a equação 2 .

$Q_{s t}=q_{s t}+\Delta H_{v}$

A entalpia de vaporização da água $\left(\Delta \mathrm{H}_{\mathrm{v}}\right)$ foi obtida a partir de tabelas de pressão de vapor e o calor isostérico liquido de adsorção $\left(\mathrm{q}_{\mathrm{st}}\right)$, através da equação 3; forma integrada da equação de Clausius-Clayperon.

$\ln \left(\frac{a_{w_{1}}}{a_{w_{2}}}\right)=\frac{-q_{s t}}{R} \cdot\left(\frac{1}{T_{1}}-\frac{1}{T_{2}}\right)$

\section{6 - Determinação da monocamada}

A umidade equivalente à monocamada $\left(\mathrm{m}_{\mathrm{o}}\right)$ foi determinada através do modelo de BET (equação 4) [3], com auxilio do aplicativo STATISTICA for Windows 5.1B.

$m=\frac{m_{o} \cdot C \cdot a_{w}}{\left(1-a_{w}\right)\left[1+(C-1) \cdot a_{w}\right]}$

onde: $\mathrm{m}$ é a umidade, $\mathrm{a}_{\mathrm{w}}$ atividade de água; $\mathrm{m}_{\mathrm{o}}$ umidade equivalente à monocamada, $\mathrm{C}$ constante relacionada com o calor de adsorção.

\section{3 - RESULTADOS E DISCUSSÃO}

\section{1 - Caracterização física e físico-química}

Os dados relativos à caracterização física e a composição das pupunhas utilizadas na obtenção da farinha são apresentados na Tabela 3. Os desvios padrões obtidos nas determinações de peso, diâmetro e comprimento do fruto foram $0,21,0,23$ e 0,12 , respectivamente. As composições em polpa, casca e caroço, foram obtidas a partir das massas totais, como mencionado no item 2.1.1.

TABELA 3. Caracterização fĩsica e composição da pupunha in natura.

\begin{tabular}{lclc}
\hline \multicolumn{2}{c}{ Caracterização física } & \multicolumn{2}{c}{ Composição } \\
Peso dos frutos $(\mathrm{g})$ & 30,6 & Polpa $(\%)$ & 72,3 \\
Diâmetro $(\mathrm{cm})$ & 3,4 & Casca $(\%)$ & 6,3 \\
Comprimento $(\mathrm{cm})$ & 3,1 & Caroço $(\%)$ & 21,4 \\
\hline
\end{tabular}

Os resultados obtidos nas análises físico-químicas das pupunhas in natura e cozidas e da farinha, são apresentados na Tabela 4.

Comparando os resultados com os apresentados nas Tabelas 1 e 2, pode-se observar algumas variações, as quais são perfeitamente justificáveis com base na utilização de uma variedade de pupunha diferente.
TABELA 4. Composição fîsico-química da pupunha e da farinha de pupunha.

\begin{tabular}{lccc}
\hline \multicolumn{3}{c}{$\mathbf{g} / \mathbf{1 0 0} \mathbf{~ g ~ d e ~ p r o d u t o ~}$} \\
\hline Componentes $^{*}$ & “in natura” & Cozida & Farinha \\
\hline Umidade & 68,5 & 64,8 & 5,3 \\
Lipídios & 1,9 & 1,9 & 4,6 \\
Proteínas & 2,4 & 1,3 & 22,7 \\
Fibras & 1,6 & 1,4 & 1,1 \\
Carboidratos & 25,1 & 30,0 & 64,7 \\
Cinzas & 0,5 & 0,6 & 1,6 \\
\hline
\end{tabular}

* Médias de duas determinações (erros relativos inferiores a 10\%)

\section{2 - Isotermas de adsorção de umidade}

Os dados de equilíbrio de adsorção obtidos para a farinha de pupunha são apresentados na Tabela 5 e representados graficamente nas Figuras 2 e 3.

As isotermas apresentam comportamento típico de isotermas do tipo III, de acordo com classificação da IUPAQ [10].

A pequena variação na umidade de equilíbrio em umidades relativas inferiores a $50 \%$ e a considerável variação, a partir deste valor, indicam que o produto expira maiores cuidados ao ser manipulado ou armazenado em ambientes com umidades relativas superiores a $50 \%$. Um aumento na umidade do produto torna-o propenso ao desencadeamento de reações indesejáveis e/ou proliferação de microrganismos.

TABELA 5. Dados de equilíbrio de adsorção para a farinha de pupunha.

\begin{tabular}{|c|c|c|c|}
\hline \multicolumn{2}{|r|}{$15^{\circ} \mathrm{C}$} & \multicolumn{2}{|r|}{$35^{\circ} \mathrm{C}$} \\
\hline $\mathbf{a}_{\mathrm{w}}$ & $\mathrm{m}\left(\mathrm{g} \mathrm{H}_{2} \mathrm{O} / 100 \mathrm{~g} \mathrm{s.s.}\right)^{\star}$ & $\mathbf{a}_{\mathrm{w}}$ & $\mathrm{m}\left(\mathrm{g} \mathrm{H}_{2} \mathrm{O} / 100 \text { g s.s.) }\right)^{\star}$ \\
\hline 0,11 & 0,27 & 0,11 & 0,20 \\
\hline 0,23 & 1,21 & 0,23 & 1,09 \\
\hline 0,33 & 1,96 & 0,32 & 1,25 \\
\hline 0,44 & 3,29 & 0,41 & 2,86 \\
\hline 0,57 & 5,35 & 0,57 & 4,69 \\
\hline 0,75 & 10,26 & 0,75 & 9,49 \\
\hline 0,88 & 17,21 & 0,84 & 17,16 \\
\hline 0,97 & 50,30 & 0,96 & 43,80 \\
\hline
\end{tabular}

* Médias de duas determinações (erros relativos inferiores a 10\%)

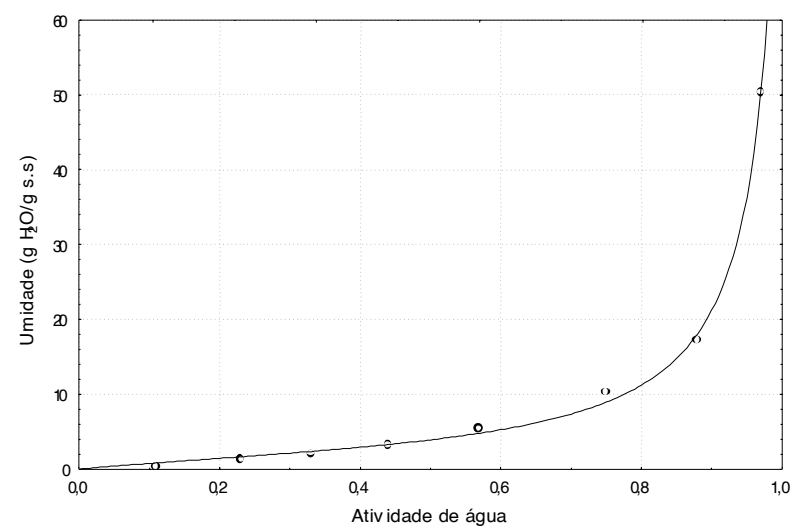

FIGURA 2. Isoterma de adsorção da farinha de pupunha a $15^{\circ} \mathrm{C}$. 


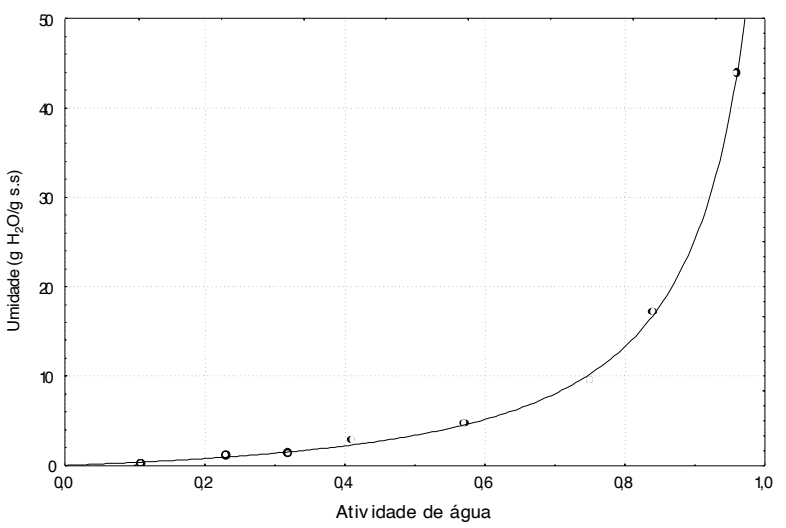

FIGURA 3. Isoterma de adsorção da farinha de pupunha a $35^{\circ} \mathrm{C}$.

A umidade de equilibrio apresentou uma sensivel diminuição $(\approx 10 \%)$, para um aumento de $20^{\circ} \mathrm{C}$ na temperatura. AYERST [2] observou variações médias de $10 \%$ na umidade de equilibrio de diversos produtos, para variações de $10^{\circ} \mathrm{C}$.

A diminuição da umidade de equilíbrio com o aumento da temperatura pode ser justificada com base no aumento da pressão de vapor da água no ar e na superficie do produto. Este aumento é maior na superficie do produto, pois a mesma apresenta maior número de moléculas de água que o ar. Maior pressão de vapor implica em maior perda de água, para que se atinja o equilibrio.

\section{3 - Ajuste do modelo de GAB}

Os parâmetros do ajuste do modelo de GAB aos dados de equilibrio são apresentados na Tabela 6. As constantes $\mathrm{m}_{\mathrm{o}}$, c e k, estão dentro da faixa encontrada por LOMAURO et al. [14, 15], para alimentos com diversas composições.

TABELA 6. Parâmetro do ajuste do modelo de GAB.

\begin{tabular}{ccccc} 
& \multicolumn{4}{c}{ Parâmetros } \\
Temperatura & $\mathbf{m}_{\mathbf{0}}$ & $\mathbf{c}$ & $\mathbf{k}$ & $\mathbf{r}$ \\
\hline $15^{\circ} \mathrm{C}$ & 2,67 & 3,11 & 0,98 & 0,9991 \\
$35^{\circ} \mathrm{C}$ & 5,12 & 0,61 & 0,94 & 0,9996 \\
\hline
\end{tabular}

Os bons ajustes da equação de GAB aos dados de equilíbrio são evidenciados pelos valores de $\mathrm{r}$ (coeficiente de regressão), apresentados na Tabela 6 , podendo ser visualizados nas representações gráficas das Figuras 2 e 3 .

\section{4 - Cálculo do calor isostérico de adsorção}

Os calores isostéricos liquidos de adsorção foram determinados em diferentes niveis de unidade, a partir dos dados de equilíbrio de adsorção. Os calores isostéricos integrais de adsorção foram obtidos acrescentando-se aos calores liquidos, o calor latente de vaporização da água $\left(\Delta \mathrm{H}_{\mathrm{v}}\right)$, na temperatura máxima de trabalho $\left(35^{\circ} \mathrm{C}\right)$, o qual é $43,5 \mathrm{~kJ} / \mathrm{mol}$. A representação gráfica dos dados está na Figura 4.

Observa-se a ocorrência de calores inferiores ao calor latente de vaporização da água, não esperado, visto que a menor quantidade de calor necessária para evapo- rar a água é o calor latente de vaporização. Os erros observados chegam no máximo a 3\%, podendo ser justificados pelo acúmulo de erros na obtenção de valores de $\mathrm{a}_{\mathrm{w}}$, em cada umidade. Estes valores foram determinados a partir das equações obtidas com os ajustes matemáticos.

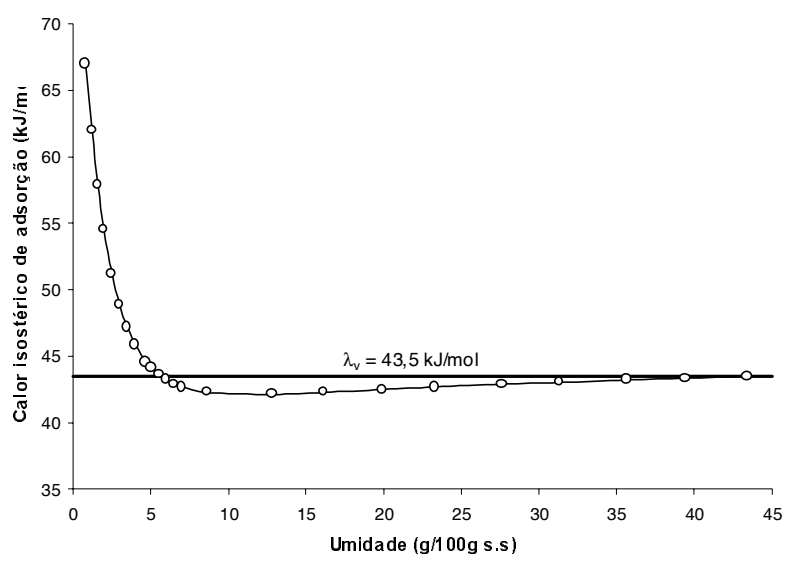

FIGURA 4. Calores isostéricos integrais de adsorção para a farinha de pupunha.

A Figura 4 evidencia que a umidade equivalente à monocamada deve ser inferior a $5 \mathrm{~g} \mathrm{H}_{2} \mathrm{O} / 100 \mathrm{~g}$ s.s., visto que só abaixo deste valor o calor isostérico integral de adsorção passa a ser maior que o calor latente de vaporização da água, indicando interações mais fortes entre moléculas de água e os sítios de superfície do produto.

Comparando as umidades de equilíbrio máximas: $50,3 \mathrm{~g} \mathrm{H}_{2} \mathrm{O} / 100 \mathrm{~g}$ s.s. para $15^{\circ} \mathrm{C}$ e $43,8 \mathrm{~g} \mathrm{H}_{2} \mathrm{O} / 100 \mathrm{~g}$ s.s. para $35^{\circ} \mathrm{C}$, com o valor de $5 \mathrm{~g} \mathrm{H}_{2} \mathrm{O} / 100 \mathrm{~g}$ s.s., constata-se que a maior parte da água é adsorvida no produto, através de condensação capilar ou outros fenômenos físicos que envolvem pequenas quantidades de energia, ou seja, interações muito fracas entre as moléculas de água e o soluto. Isso indica a baixa higroscopicidade da farinha de pupunha, a qual é confirmada pela aglomeração do produto, que ocorreu para o mais alto nivel de umidade relativa, ao qual foi exposto o produto $\left(97 \%\right.$ a $15^{\circ} \mathrm{C}$ e $96 \%$ a $\left.35^{\circ} \mathrm{C}\right)$.

\section{5 - Determinação da monocamada}

As umidades equivalentes à monocamada $\left(\mathrm{m}_{\mathrm{o}}\right)$, determinadas por BET, são apresentadas na Tabela 7, juntamente com os coeficientes de regressão (r).

Os valores de $m_{0}$ nas duas temperaturas de trabalho são inferiores a $5 \mathrm{~g} \mathrm{H}_{2}^{\circ} \mathrm{O} / 100 \mathrm{~g}$ s.s., como havia sido predito através dos calores isostéricos de adsorção. Observa-se, ainda, um aumento no valor de $\mathrm{m}_{\mathrm{o}}$ com a temperatura. Apesar deste comportamento não ser muito comum, dois mecanismos podem ser utilizados para justificá-lo. Um aumento na temperatura pode: provocar modificações na estrutura física do produto, disponibilizando um maior número de sítios ativos com afinidade por moléculas de água, ou um aumento na solubilidade de solutos intrinse$\cos$ ao produto, fazendo com que um maior número de moléculas de água fiquem retidas na monocamada. A solubilidade é o resultado de um fenômeno de solvatação. 
TABELA 7. Umidades de equilíbrio equivalentes à monocamada.

\begin{tabular}{cccc}
\hline & $15^{\circ} \mathrm{C}$ & \multicolumn{2}{c}{$35^{\circ} \mathrm{C}$} \\
\hline $\mathbf{m}_{\circ}$ & $\mathbf{r}$ & $\mathbf{m}_{\circ}$ & $\mathbf{r}$ \\
\hline 1,56 & 0,9891 & 1,83 & 0,9845 \\
\hline
\end{tabular}

Comparando os valores de $\mathrm{m}$ determinados pelas equações BET (Tabela 7 ) e GAB (Tabela 6), observa-se que o comportamento frente à temperatura se manteve, mas os valores obtidos por GAB são superiores aos de $\mathrm{BET}$ e se aproximam mais do valor de $\mathrm{m}_{\mathrm{o}}$ sugerido pelo comportamento dos calores de adsorção.

A equação BET não fornece bons resultados na determinação de $\mathrm{m}_{\mathrm{o}}$ para produtos com isotermas características do Tipo III [24], que é o caso do produto. Os coeficientes de regressão podem ser utilizados como parâmetro de comparação para justificar, de fato, os melhores ajustes para a equação GAB. Desta forma os valores de $m_{0}$ determinados por $\mathrm{GAB}$ apresentam uma maior confiabilidade.

\section{4 - CONCLUSÕES}

- A farinha de pupunha pode ser obtida por um processo de secagem simples da polpa da pupunha in natura, mantendo grande parte de seus constituintes nutricionais originais, o que indica a viabilidade de sua produção.

- Os dados de equilíbrio de adsorção, obtidos para a farinha de pupunha, indicam que o produto apresenta baixa higroscopicidade.

- A umidade de equilíbrio diminui com o aumento da temperatura, indicando mudanças nos mecanismos de interação da água com os sitios de superficie do produto, ou nos próprios sítios.

\section{5 - REFERENCIAS}

[1] ASSOCIATION Official Analytical Chemists. Official methods of analytical of the Association of Official Analytical Chemists, 4.ed., Virginia, 1984. 1094p.

[2] AYERST, G. Determination of the water activity of some hygroscopic food materials by a dew-point method. Journal of the Science of Food and Agriculture, v. 16, n.2, p.71-78, 1965.

[3] BRUNAUER, S.; EMMET, P.H.; TELLER, E. Adsorption of gases in multimolecular layers. Journal American Chemical Society, v.60, p.309-319, 1938.

[4] CARVALHO, S.M.L. Determinação de isotermas de adsorção da farinha d'água obtida de mandioca (Manihot esculenta). Belém, 1994. Trabalho de Conclusão de Curso (Especialização em Tecnologia de Alimentos) - Departamento de Engenharia Química, Universidade Federal do Pará (UFPA).

[5] Clement, C.R. Domestication of the Pejibaye Palm (Bactris gasipaes): Past and Present. Advances in Economic Botany, n.6, 1988.

[6] CLEMEnT, C.R. Pupunha: uma árvore domesticada. Ciência Hoje, volume especial, dezembro, 1991.

[7] GOIA, C.H.; ANDRADE, J.S.; ARAGÃO, C.G. Composição química da farinha de pupunha. Revista de Química Industrial, v.2, n.2, p.48-54, 1993.
[8] GÓIS, V.A. de. Comportamento higroscópico do mamão liofilizado com vistas ao estabelecimento do seu potencial de "caking". São Paulo, 1981, 114p. Dissertação (Mestrado) - Escola Superior de Agricultura de Lavras.

[9] INSTITUTO ADOLF LUTZ. Normas analíticas do Instituto Adolfo Lutz, métodos químicos e físicos para análises de alimentos, 3.ed., vol.1, São Paulo, 1985.

[10] INTERNATIONAL Union of Pure and Applied Chemistry IUPAQ. Reporting physisorption data for gas/solid systems. Pure and Applied Chemistry, v.57, n.4, p.603-619, 1985.

[11] KAREL, M. Water activity and food preservation. In: FENNEMA, O.R., ed. Principles of food science; physical principles of food preservation. New York, Marcel Dekker, 1975. v.4, p.237-265.

[12] KIMURA, I.A.; MAEDA, M. Determinação de isotermas de adsorção de umidade: influência da concentração da solução e da variação no volume de recipiente. Boletim da Sociedade Brasileira de Ciência e Tecnologia de Alimentos, v.27, n.2, p.79-87, 1993.

[13] LANDROCK, A.H.; PROCTOR, B.E. A new graphical interpolation method for obtaining humidity equation data, with special reference to its role in food packaging studies. Food Technology, v.5, n.8, p.332-337, 1951.

[14] LOMAURO, C.J.; BAKSHI, A.S.; LABUZA, T.P. Evaluation of food moisture sorption isotherm equations. Part I : fruit, vegetable and meat products. Lebensmittel Wissenschaft und Technologie, v.18, n.2, p.111-117, 1985.

[15] LOMAURO, C.J.; BAKSHI, A.S.; LABUZA, T.P. Evaluation of food moisture sorption isotherm equations. Part II: milk, coffee, tea, nuts, oilseeds, spices and starchy foods. Lebensmittel Wisseschaft und Technologie, v. 18, n.2, p.118-124, 1985.

[16] MAROULIS, Z.B.; TSAMI, E.; ARINOS-KOURIS, D.; et al. Application of the GAB model to the sorption isotherms for dried fruits. Journal of Food Engeneering, 1988.

[17] MORA-URPI, J. Diversidad genética en pejibaye [Bactris(guilielma) gasipaes Kunth] : Origem y domesticación: p:21-30. In: J. Mora- Urpi, et al. IV Congresso Internacional sobre biología, agronomía e industrialización del Pijuayo. Editorial de la Universidade de Costa Rica, San José, Costa Rica, 1993.

[18] PENA, R.S. Comportamento higroscópico do pó de guaraná (Paulinia cupana) obtido em secador por nebulização. Belém, 1994, 120p. Dissertação (Mestrado em Engenharia Química) - Universidade Federal do Pará (UFPA).

[19] PENA, R.S.; RIBEIRO, C.C.; GRANDI, J.G. Levantamento de isoterma de adsorção de umidade a $25^{\circ} \mathrm{C}$, do guaraná (Paullinia cupana) em pó, obtido por atomização em spray dryer. Anais do Congresso Brasileiro de Engenharia Química, 10. 1994. v.2. p.1383-1388.

[20] PRIOR, B.A.J. Measurement of water activity in food : A Review. Journal of Food Protection, v.42, n.8, p.668-674, 1969.

[21] QUAST, D.G. Físico-Química da água em alimentos desidratados. Boletim do Centro Tropical de Pesquisas e Tecnologia de Alimentos, n.13, p.1-25, 1968.

[22] ROCKLAND, L.B. A new treatment of hygroscopic equilibria: application to walnuts (Juglans regia) and other foods. Food Research, v.22, p.604-628, 1957.

[23] ROCKLAND, L.B. Saturated salt solutions for static control of relative humidity between $5^{\circ} \mathrm{C}$ and $40^{\circ} \mathrm{C}$. Analytical Chemistry, v.32, n.10. p.1375-1376, 1960.

[24] SALWIN, H. Moisture levels required for stability in dehydrated foods. Food Technology, v.17, n.9, p.1114-1121, 1963.

[25] TEIXEIRA NETO, R.O; DENIZO, N.; QUAST, D.G. Atividade de água em alguns alimentos de teor intermediário de umidade. II. Coletânea do Instituto de Tecnologia de Alimentos, v.7, p.191-207, 1976. 